\title{
Near-infrared dye having a large ultrafast third-order susceptibility
}

\author{
C. Maloney and W. Blau \\ Laser Group, Department of Pure and Applied Physics, Trinity College, Dublin 2, Ireland
}

K. H. Drexhage

Physikalisch-Chemisches Institut der Universität Siegen, D-5900 Siegen, Federal Republic of Germany

Received January 21, 1986; accepted May 1, 1986

\begin{abstract}
Efficient degenerate four-wave mixing of 160-psec infrared light pulses at $\lambda=1.064 \mu \mathrm{m}$ in solutions of a new dye that absorbs between 1.0 and $1.6 \mu \mathrm{m}$ is reported. A Kerr-like third-order susceptibility of $\left.\chi_{x y y x}^{(3)}=8 \pm 3\right) \times 10^{-12}$ esu with a decay time of less than 50 psec is observed in various solvents.
\end{abstract}

Degenerate four-wave mixing (DFWM) is a nonlinear technique that is widely used as a spectroscopic tool ${ }^{1}$ and also as a method of producing optical phase conjugation. ${ }^{2}$ Its potential application to optical data and image processing ${ }^{3,4}$ has stimulated extensive research with the aim of developing materials having high nonlinear susceptibilities and fast response and decay times.

In recent years organic dyes have been much used as nonlinear media. ${ }^{5-12}$ The dyes have usually been dissolved in organic solvents ${ }^{5-10}$ or hosted in transparent organic matrices. ${ }^{11,12}$ In dye-gelatin films large effective nonlinearities have been observed, paired, however, with a very slow decay time (several seconds) of the nonlinear excitation. ${ }^{11,12}$ In solution, saturable $a b-$ sorption ${ }^{9,10}$ and thermally induced refractive-index changes $^{5-8}$ have been found to give rise to nonlinearities with characteristic decay times ranging from $10^{-9}$ to $10^{-5}$ sec.

We have investigated the suitability of a recently synthesized infrared-absorbing dye as a nonlinear medium for DFWM with picosecond near-infrared light pulses. The generation of a population grating is eliminated by using orthogonally polarized pump and probe beams. ${ }^{13,14}$ Therefore neither saturable absorption nor thermally induced refractive-index gratings can contribute to the signal. Thus the only possible interaction is an optical Kerr effect, which may be either orientational or electronic (displacement of the $\pi$-electron cloud) in origin. ${ }^{1}$

The chemical structural formula of the dye used is shown in Fig. 1 together with its absorption spectrum in a dilute $o$-dichlorobenzene solution. The $S_{0}-S_{1}$ absorption band extends from 0.9 to $1.6 \mu \mathrm{m}$. A compound with similar chemical and structural properties (S501) has been reported as a laser dye that generates amplified spontaneous emission in the region of 1.8 $\mu \mathrm{m} .{ }^{15}$

Solutions of the dye in 1,2-dichloroethane, chlorobenzene, $o$-chlorophenol, and $o$-dichlorobenzene were contained in $L=2-\mathrm{mm}$-thick fused-silica cuvettes. The sample transmissions were adjusted to give opti- mum DFWM signal and were in the range $T=0.54$ to $T=0.74$, corresponding to absorption coefficients of $\alpha$ $=135 \mathrm{~m}^{-1}$ to $\alpha=295 \mathrm{~m}^{-1}$ and dye concentrations of $(1.2 \pm 0.4) \times 10^{-4} \mathrm{~mol} /$ liter.

For nonlinear-optical experiments, liquid solutions have certain advantageous properties that set them apart from solids in that they are generally self-healing and tend to have high damage thresholds. In addition, good optical quality is readily attainable, and the optical parameters may easily be modified.

The dye was found to be most stable when dissolved in substituted benzene derivatives, such as $o$-dichlorobenzene and $o$-chlorophenol. In these solvents the samples had a usable lifetime of several weeks. No significant saturation of the dye transmission was apparent in any of the solutions at the laser intensities used-up to $1 \mathrm{GW} / \mathrm{cm}^{2}$.

The experiments were performed with 160-psec pulses generated by an amplified mode-locked $\mathrm{Nd}^{3+}: \mathrm{YAG}$ laser system. The experimental arrangement is shown in Fig. 2. Three pulses (with energies

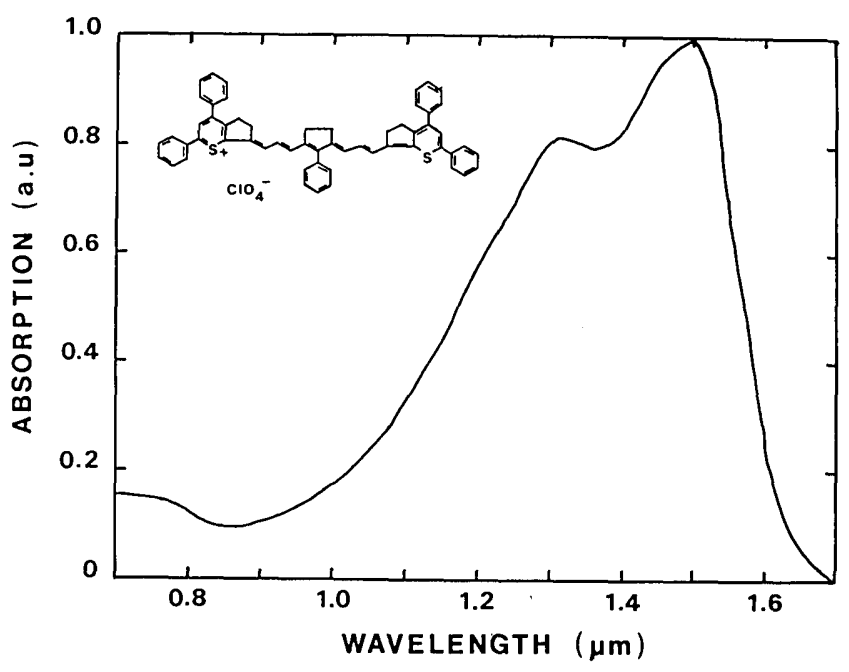

Fig. 1. Chemical structural formula of the dye used and its absorption spectrum in $o$-dichlorobenzene. 


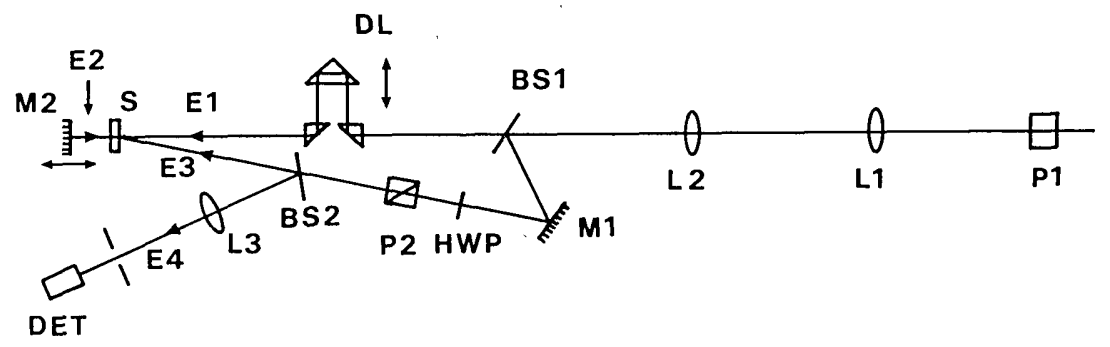

Fig. 2. Experimental arrangement for degenerate four-wave mixing using retroflection geometry.

$E 1, E 2$, and $E 3)$ are incident upon the sample S. Two intense pump pulses $E 1$ and $E 2$ are counterpropagating. A retroreflection geometry is employed in which $E 2$ is derived from $E 1$ after transmission through the sample and reflection from the mirror M2. A less intense probe pulse, $E 3$, is incident at an angle $8^{\circ}$ to $E 1$ $(E 3 \simeq 0.09 E 1)$. A delay line $\mathrm{DL}$ is used to ensure that the three pulses arrive simultaneously at the sample. The polarization of $E 3$ is rotated with a half-wave plate HWP and confirmed with a polarizer P2. The signal pulse $E 4$ retraces the path of $E 3$. Part of $E 4$ is split off from $E 3$ by a pellicle beam splitter BS2 and is spatially filtered by a lens aperture combination in order to discriminate against scattered background light. Its energy $E 4$ is measured by a Si photodiode DET.

The beam is telescoped by lenses L1 and L2 in order to increase the intensity up to $I_{1} \leq 1 \mathrm{GW} / \mathrm{cm}^{2}$. The spot sizes of the pump and probe beams at the sample were equal ( $1 / e$ radii $r_{0}=0.4 \mathrm{~mm}$ ) with Gaussian beam profiles. This arrangement facilitates measurement of the decay time of the nonlinear excitation, which may be probed by displacing mirror M2 and hence delaying the incidence of $E 2$.

The intensity of the pump pulse $E 1$ was determined by measuring its energy transmission through the mode-locking dye A9860. ${ }^{16}$ A9860 is a well-characterized saturable absorber for the laser wavelength $\lambda=$ $1.064 \mu \mathrm{m}$. Thus, by monitoring the transmission of the pump pulse through a 1-mm-thick cell filled with an A9860-1,2-dichloroethane solution (low-light-level transmission $T_{0}=0.173$ ) the intensity of the pulse can be determined from the bleaching curve as described in Ref. 16. This provides a convenient and simple routine check of the laser intensity without the need to measure energy, pulse width, and beam cross section simultaneously.

The dependence of the phase-conjugate peak reflectivity, $R_{\mathrm{pc}}=I_{4} / I_{3}$, on the incident pump peak intensity $I_{1}$ was measured. 'It was found to be quadratic, as shown in Fig. 3, for a 1,2-dichloroethane solution. This is in agreement with theoretical predictions. ${ }^{1}$ A peak reflectivity of 0.27 was observed at a pump intensity of $0.8 \mathrm{GW} / \mathrm{cm}^{2}$. The third-order nonlinear susceptibility may be calculated from ${ }^{17}$

$$
\chi^{(3)}=\frac{4 c^{2} n^{2} \epsilon_{0} \alpha \sqrt{R_{\mathrm{pc}}}}{3 \omega I_{1} T(1-T)},
$$

where $n$ denotes the refractive index of the nonlinear medium, $\omega$ the angular frequency, $c$ the velocity of light in vacuum, and $\epsilon_{0}$ the dielectric constant. The remaining symbols are explained in the text.

A DFWM signal was not detected for a neat 1,2dichloroethane sample. For the other solvents mentioned, however, considerable contributions to the signal were measured. The nonlinear susceptibility $\chi^{(3)}$ of the dye may be evaluated directly, as the sign of $\chi^{(3)}$ is the same for the dye and solvents-both being Kerr susceptibilities. The measured values of $\chi^{(3)}$ for the four dye solutions are shown in Table 1 together with those measured for the neat solvents. For each of the solutions, with the exception of the 0 -chlorophenol, a value of $\chi_{x y y x}^{(3)}=(8 \pm 2) \times 10^{-12}$ esu or $\chi_{x y y x}^{(3)}=(1.2 \pm$ $0.25) \times 10^{-19} \mathrm{~m}^{2} / \mathrm{V}^{2}$ is calculated by using Eq. (1). ${ }^{19}$ The relatively small value of the $o$-chlorophenol solution may originate from a strong solvent-solute interaction, which is also observed by a change in the absorption spectrum of the dye. These results indicate that the nonlinearity is electronic in origin. ${ }^{20}$

In order to investigate the temporal behavior of the nonlinearity, the DFWM signal energy $E 4$ was measured as a function of second pump-pulse $E 2$ delay

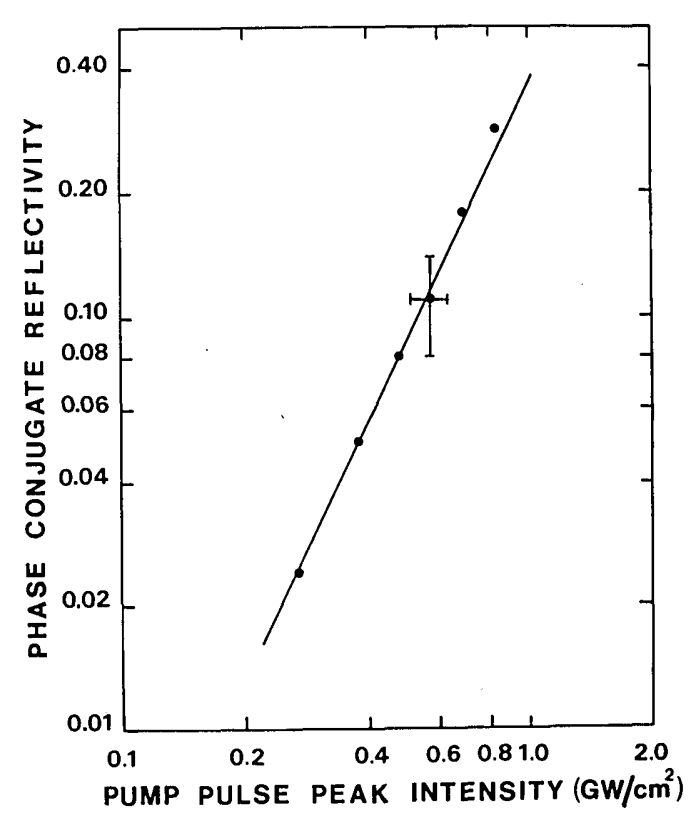

Fig. 3. Dependence of the phase-conjugate peak reflectivity $R_{\mathrm{pc}}=I_{4} / I_{3}$ on the first pump-pulse intensity $I_{1}$ for a dye1,2-dichloroethane solution, with an absorption coefficient $\alpha$ $=280 \mathrm{~m}^{-1}$. 
Table 1. Comparison of Third-Order Nonlinear Susceptibilities $\chi_{x y y x}^{(3)}$ for the Dye in Various Solvents with $\mathrm{CS}_{2}$

\begin{tabular}{lccc}
\hline \multicolumn{1}{c}{ Solvent } & $\chi_{\text {total (esu) }}^{(3)}$ & $\chi_{\text {solvent (esu) }}^{(3)}$ & $\chi_{\text {dye (esu) }}^{(3)}$ \\
\hline o-Dichlorobenzene & $(11 \pm 2) \times 10^{-12}$ & $(3 \pm 1) \times 10^{-12}$ & $(8 \pm 3) \times 10^{-12}$ \\
o-Chlorophenol & $(7 \pm 2) \times 10^{-12}$ & $(4 \pm 1) \times 10^{-12}$ & $(3 \pm 2) \times 10^{-12}$ \\
Chlorobenzene & $(10 \pm 2) \times 10^{-12}$ & $(2 \pm 1) \times 10^{-12}$ & $(8 \pm 3) \times 10^{-12}$ \\
1,2-Dichloroethane & $(8 \pm 2) \times 10^{-12}$ & $<5 \times 10^{-13}$ & $(8 \pm 2) \times 10^{-12}$ \\
\multicolumn{1}{c}{ Carbon disulfide } & $7.6 \times 10^{-14}{ }^{a}$ & & \\
\hline
\end{tabular}

a Picosecond value from Ref. 20 .

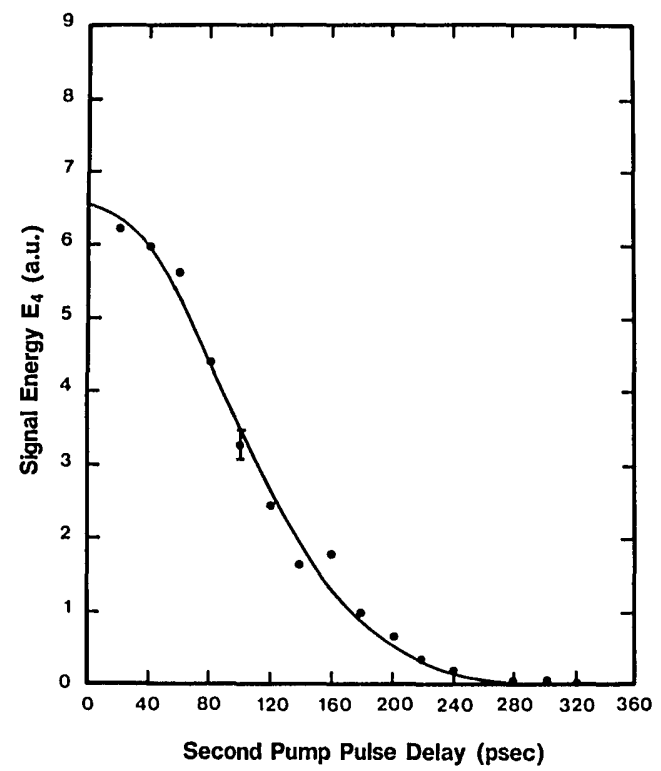

Fig. 4. Dependence of the phase-conjugate signal energy $E 4$ on second pump-pulse delay for a dye-o-chlorophenol solution.

(Fig. 4). The response time of the sample is instantaneous. The signal decays with the intensity autocorrelation function

$$
S\left(t_{d}\right)=\int I^{2}(t) I\left(t-t_{d}\right) \mathrm{d} t
$$

of the pulse. This provides evidence that the decay time of the nonlinearity is considerably shorter than the pulse width (decay time $\lesssim 50$ psec). Assuming that the temporal profile of the pulses is Gaussian, a half-width (FWHM) of the autocorrelation curve of 195 psec can be calculated that is in agreement with the measured value $200 \pm 20$ psec. Further evidence for the fast decay time is given by the fact that the curve does not follow the coherence autocorrelation function, as would be expected for a slow nonlinearity. An orientational Kerr effect can be ruled out, as the orientational relaxation time for such large molecules as used here is greater than 200 psec. $^{21}$

We have reported picosecond DFWM in a new infrared-absorbing dye. A high third-order nonlinear susceptibility $\chi_{x y y x}^{(3)}=(8 \pm 2) \times 10^{-12}$ esu was measured using cross-polarized pump and probe beams. The origin of the nonlinearity is attributed to an electronic Kerr effect with ultrafast decay time ( $<50 \mathrm{psec})$. Although the results presented here were obtained at a single laser wavelength, similar behavior should be observed at other wavelengths. Most significantly, the dye should be useful throughout the wavelength range of interest for optical communications as well as at the operation wavelength of the iodine laser, which is a possible candidate for laser fusion.

Financial support by British Petroleum Venture Research, Guinness and Company Limited, and the Investment Bank of Ireland is gratefully acknowledged.

\section{References}

1. J. F. Reintjes, Nonlinear Optical Parametric Processes in Liquids and Gases (Academic, Orlando, Fla., 1984), p. 359.

2. R. A. Fisher, ed., Optical Phase Conjugation (Academic, New York, 1983).

3. J. O. White and A. Yariv, Appl. Phys. Lett. 37, 5 (1980).

4. T. R. O'Meara and A. Yariv, Opt. Eng. 21, 237 (1982).

5. G. Martin and R. Hellwarth, Appl. Phys. Lett. 34, 371 (1979).

6. J. O. Tocho, W. Sibbett, and D. J. Bradley, Opt. Commun. 34, 12 (1980).

7. J. O. Tocho, W. Sibbett, and D. J. Bradley, Opt. Commun. 37, 67 (1981).

8. R. G. Caro and M. C. Gower, Opt. Lett. 6, 557 (1981).

9. B. J. Feldman, R. A. Fisher, and S. L. Shapiro, Opt. Lett. 6, 84 (1981).

10. E. I. Moses and F. Y Wu, Opt. Lett. 5, 64 (1980).

11. Y. Silberberg and I. Bar-Joseph, Opt. Commun. 39, 62 (1981).

12. H. Fujiwara and K. Nakagawa, Opt. Commun. 55, 386 (1985).

13. A. L. Smirl, T. F. Boggess, B. S. Wherett, G. P. Pengman, and A. Miller, Phys. Rev. Lett. 49, 933 (1982).

14. W. M. Dennis, W. Blau, and D. J. Bradley, Appl. Phys. Lett. 47, 200 (1985).

15. A. Seilmeier, H. J. Polland, T. Elsässer, W. Kaiser, M. Kussler, N. J. Marx, B. Sens, and K. H. Drexhage, in Laser Spectroscopy VI, H. P. Weber and W. Lüthy, eds. (Springer-Verlag, Berlin, 1983), p. 424.

16. A. Penzkofer, D. Von der Linde, and A. Laubereau, Opt. Commun. 4, 377 (1972).

17. R. G. Caro and M. C. Gower, IEEE J. Quantum Electron. QE-18, 1375 (1982).

18. K. C. Rustagi and J. Ducuing, Opt. Commun. 10, 258 (1974).

19. Conversion factor: $\chi_{(\text {esu })}^{(3)}=\left(9 \times 10^{8}\right) / 4 \pi \chi^{(3)}\left(\mathrm{m}^{2} / \mathrm{V}^{2}\right)$.

20. N. Phu Xuan, J. L. Ferrier, J. Gazengel, and G. Rivoire, Opt. Commun. 51, 433 (1984).

21. H. E. Lessing and A. von Jena, in Laser Handbook, M. L. Stitch, ed. (North-Holland, Amsterdam, 1979), Vol. III, p. 753 . 